\title{
Recent results of the ANTARES neutrino telescope
}

\author{
Juan de Dios Zornoza* \\ IFIC, Instituto de Física Corpuscular (CSIC - Univ. de Valencia), Ed. Institutos de Investigacón, \\ AC22085, E46071, Valencia, Spain \\ E-mail: zornoza@ific.uv.es
}

\begin{abstract}
The ANTARES collaboration completed in 2008 the construction of the largest neutrino telescope in the Northern hemisphere. Neutrinos, being neutral, stable and weakly-interacting have unique advantages with respect to other more traditional probes to study the high energy Universe. The scientific scope of ANTARES is very broad, including the observation of astrophysical neutrino sources, the indirect detection of dark matter and more exotic searches likes monopoles or nuclearites. The data gathered in the last years has provided a rich output. In this talk I will overview the most relevant results: the skymap of the Southern neutrino sky, with the best flux limits up to date for most of that region; the limits for dark matter searches in the Sun, which are interpreted in the frameworks of MSSM and mUED; an extensive multi-messenger program, which includes search for correlations with GRBs, micro-quasars, UHE cosmic rays of Auger and grativational waves; searches for monopoles and nuclearites, and the observation of neutrino oscillations (the first time this is measured with a neutrino telescope).
\end{abstract}

36th International Conference on High Energy Physics,

July 4-11, 2012

Melbourne, Australia

\footnotetext{
* Speaker.

${ }^{\dagger}$ On behalf of the ANTARES collaboration
} 


\section{Introduction}

Neutrino astronomy has become a mature field in Astrophysics and Particle Physics. There are specific adavantages of neutrinos as cosmic probes when compared with traditional alternatives as photons or cosmic rays. Photons, in particular at high energies, interact with radiation and matter, which severely limits their range. Cosmic rays, in addition to interact with matter and radiation, are deflected by galactic and extra-galactic magnetic fields, so their directional information is lost. Neutrinos interact weakly and are neutral and stable. In order to take advanges of these properties, we need to build very large detectors.

The scientific goals of neutrino telescopes are numerous. The main objective is to understand the origin of cosmic rays. As mentioned before, cosmic rays are deflected by magnetic fields and this makes very difficult to pin-point the sources emitting them, even after one century of the discovery of their extra-terrestrial origin and many observations with photons and cosmic rays themselves. Another important issue is to prove/disprove the models which explain the gamma rays observed from several souces as being produced by hadronic mechanisms. An alternative explanation would be the the lepnotic origin, but neutrinos are not produced in this case. Indirect detection of dark matter is also a major goal of neutrino telescopes. As a matter of fact, there are particular advantages in the search for dark matter with neutrino telescopes, the most sensitive experiments for the WIMP-proton elastic scattering spin dependent cross section.

The structure of the paper is as follows. In Section 2, the detector is described. Section 3 shows the results from searches for steady cosmic neutrino sources. The analyses on transient sources are explained in Section 4. Section 5 concerns the analysis looking for diffuse cosmic fluxes. The results from dark matter searches are explained in Section 6. Other analysis are briefly described in Section 7. Finally, the conclusions are summarized in Section 8.

\section{The ANTARES telescope}

The ANTARES collaboration completed in 2008 the construction of the first deep-sea neutrino telescope [10]. The operation principle of this detector is as follows. A three-dimensional array of photomultiplier tubes (PMTs) gathers the Cherenkov light induced by relativistic muons produced in the CC interactions of high-energy neutrinos with the matter in or close to the detector. Other signatures are also possible, like the showers produced in the NC interactions of all the neutrino flavors or in the CC interactions of electron or tau neutrinos. The information of the time and position of the Cherenkov hits can be used to reconstruct the original neutrino direction with an accuracy of a few tenths of degree at energies above $10 \mathrm{TeV}$.

The ANTARES detector is installed in the Mediterranean Sea, at $40 \mathrm{~km}$ of Toulon, in the French coast. It consists of 885 PMTs, grouped in triplets and distributed along 12 lines anchored to the sea bottom. The length of these lines is $450 \mathrm{~m}$ and the separation between lines is $60-75 \mathrm{~m}$. The lines are conntected to a junction box, which is link to the shore station in La Seyne-sur-Mer (France). Other relevant elements of the detector are the system of hydrophones to monitor the shape of the line and which allows for a resolution of $15 \mathrm{~cm} \mathrm{[4]} \mathrm{and} \mathrm{the} \mathrm{optical} \mathrm{beacon} \mathrm{system}$ which offers a timing resolution at the level of $1 \mathrm{~ns}$ [8]. 


\section{Point source search}

The search for cosmic point sources is one of the main goals of ANTARES. Accumulations of events above what is expected from only background are looked for in the whole sky and also in particular directions where good neutrino candidates exist. The energy information is also used, since the spectrum of cosmic signal is expected to be harder than that of background. As in other analysis, the sources of background are the atmospheric muons and atmospheric neutrinos produced after the interactions of cosmic rays in the atmosphere. This background is calculated directly from scrambled data, although Monte Carlo simulations are also done in order to check that the detector is well under control. The simulation of the signal and the response of the detector are based on Monte Carlo simulation.

The results presented here correspond to the period 2007-2010 [7] (the results of a first search, using 2007-2008 data are described in [1]). The total live time is 813 days, including 183 days of the period when only 5 lines were connected.

The selection of events is based in the following criteria. Only up-going events are selected, in order to filter out most of the atmospheric muon background, since muons cannot traverse the Earth. Still, many down-going events are mis-reconstructed as up-going (a small fraction of the total number of down-going muons, but important given the large flux involved). However, most of these events can be rejected by requiring a good quality of in the reconstruction fit. Moreover, the estimated error in the reconstructed track is also used, by requiring it to be less that one degree. The final sample of events contains 3058 neutrino candidates. Simulation predicts the about $85 \%$ of them are atmospheric neutrinos and the rest, atmospheric muons. The angular resolution for a cosmic component after these quality cuts and assuming and energy spectrum $E^{-2}$ is estimated to be $0.46 \pm 0.10$ degrees.

As mentioned before, the search is done using two different procedures. First, a scan is done looking for accumulations of events in any part of the sky. Figure 2 (left) shows the sky map of the p-values before trial factor corrections. The most signal-like cluster is found in $(\alpha, \delta)=$ $\left(-46.5^{\circ},-65.0^{\circ}\right)$, where five events are found within a one degree around. This corresponds to a $2.2 \sigma$ significance (two-sided). Second, the location of 51 sources which are good candidate to emmit neutrinos are specifically looked at. No statistically significant excess has been found, being HESS J1023-575 the most signal-like case, with a p-value of 41\%. The limits for these sources are shown in Figure 2 (right).

\section{Transient sources}

For those sources in which emission is expected to happen in specific time windows, the background can be further rejected, improving the sensitivity. This includes catastrophic events like GRBs or flaring objects like blazars or micro-quasars. In the GRB analysis 40 bursts detected in 2007 have been investigated looking for correlations between neutrinos and gamma emission, without a positive case. In the blazar analysis [2], 10 flares detected in 2008 have been analyzed. In nine of them (PKS0208-512, AO0235+164, PKS1510-089, 3C273, 3C279, 3C454.3, OJ287, PKS0454-234, Wcomae and PKS2155-304), no correlated event has been found. For 3C279, one event is found in correlation. The p-value after correction for trial factor is $10 \%$. In the micro- 

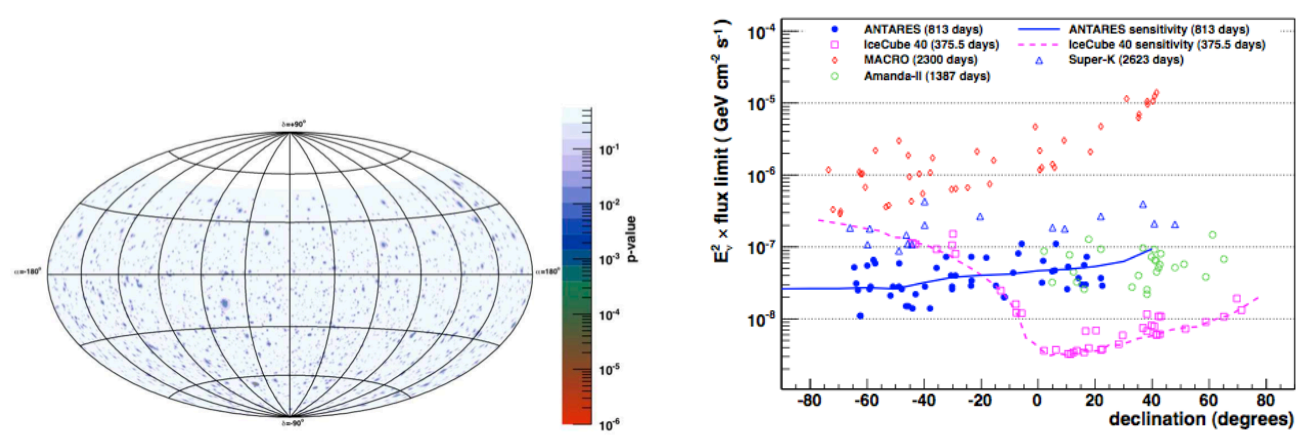

Figure 1: Left: Skymap in equatorial coordinates of p-values obtained in the all-sky search. The trial factor correction is not included in the values shown in the figure. Right: Upper limits in the neutrino flux $(90 \%$ c.1.) set by ANTARES with 2007-2010 data. The sensitivity (average upper limit) is also shown. Other limits set by different experiment are also shown for reference. An $\mathrm{E}^{-2}$ spectrum is assumed.
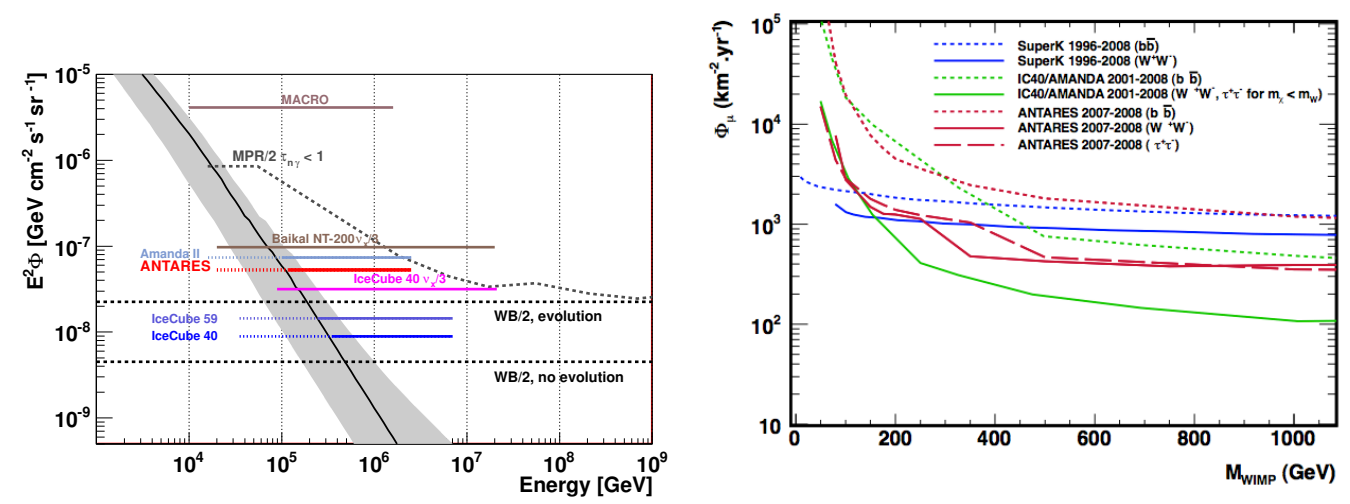

Figure 2: Left: Upper limits at $90 \%$ c.l. for the diffuse flux contribution of neutrinos and anti-neutrinos. The grey band represent the expected atmospheric neutrino flux. Its width shows the dependence of the atmospheric flux with the zenith angle. Right: Limits in the neutrino flux (90\% c.l.) from the Sun for several channels in the CMSSM and mUED frameworks. (Preliminary).

quasar analysis, 6 flares detected in 2007-2012 have been analyzed (Circinus X-1, GX339-4, H 1743-322, IGRJ17091-3624, Cygnus X-1, Cygnus X-3), with no correlation found.

\section{Diffuse fluxes}

In the search for point sources, it is assumed that the neutrino flux is dominated by a single or a few sources. It could rather be that the flux is better described as the sum of many unresolved sources. If this is the case, a better approach would be to integrate the whole signal (diffuse flux) and look for and excess with respect to the background. In this case, the information from the position is lost, but we still can use the fact that the expected spectral index for the cosmic signal is harder (typically $E^{-2}$ ) than for the background $\left(E^{-3.7}\right)$. Figure 3 (left) shows the results obtained using this strategy. Since no excess has been found, a limit to the diffuse cosmic neutrino flux is obtained. 


\section{Dark matter}

The search for dark matter is another important goal of the ANTARES experiment. One of the best sources to look at in the Sun. If dark matter is made of WIMPs (Weakly Interacting Massive Particles), they would accumulate in the Sun after scattering and become gravitationally trapped. If they are Majorana particles, they would self-annhilate and (typically after the decay of the secondaries) produce neutrinos. One of the advantages of the search in the Sun is that a potential signal would be very clean, in the sense that no other astrophysical explanation sounds likely, contrary to the case of "hints" observed by gamma detectors in the Galactic Center or by cosmic ray experiments. Moreover, neutrino telescopes give the best sensitivity to the spin-dependent cross section for WIMP proton elastic scattering. In the analysis presented here, no excess has been found in the direction of the Sun in the 2007-2008 data. Figure 3 (right) shows the neutrino flux limits from the Sun for several channels in the in the CMSSM and mUED frameworks. More details can be found in a specific contribution in this conference [13].

\section{Other searches}

Other analysis carried out by ANTARES are summarized here:

- Fermi bubbles: Data from Fermi-LAT have revealed two large almost spherical structures located above and below the Galactic plane, close to the Galactic Centre, which have been dubbed Fermi Bubbles. These observations indicate gamma emission with a hard an uniform spectrum. There are models which explain these structures as a consequence of hadronic acceleration. A on/off source search has found 75 events in the on-source region, whereas $90 \pm 5$ (stat) \pm 3 (sys) were expected from background. This excludes the fully hadronic scenario with no cut-off [11].

- Gravitational waves: Common emission of gravitational waves and neutrinos is predicted in several catastrophic astrophysical events. Data of 2007 of VIRGO. LIGO and ANTARES have been used to look for such correlations [6].

- Magnetic monopoles: Magnetic monopoles are predicted in spontaneously broken gauge theories and would be seen in ANTARES as very bright (they would yield $\sim 8500$ times the light of a muon) and slowly moving events. Flux limits have been set at $1-9 \times 10^{-17} \mathrm{~cm}^{-2}$ $\mathrm{s}^{-1} \mathrm{sr}^{-1}$, which is a factor three better than searches by other experiments [3].

- Nuclearites: Nuclearites are stable, massive lumps of strange quark matter particles. They would be seen as slowly moving downgoing events. Flux limits at $1-5 \times 10^{-17} \mathrm{~cm}^{-2} \mathrm{~s}^{-1} \mathrm{sr}^{-1}$ have been set for nuclearite masses in the range $10^{14}-10^{17} \mathrm{GeV}$ using 2007-20078 data [12].

- Neutrino oscillations: ANTARES detector does not aim for oscillations measurements and therefore its sensitivity cannot compete with other experiments specifically designed for such aim. Still, oscillations have been observed by ANTARES, with results compatible with the other experiments [5]. 


\section{Conclusions}

The ANTARES collaboration successfully completed in 2008 the first deep-sea neutrino detector. Located in the Northern Hemisphere, it has a full visibility of the Galactic Center and most of the Galactic plane. Being installed in water, it has an unsurpassed angular resolution. The data collected after more than five years of operation has produced a rich scientific output, including results on steady point cosmic sources, like the first map of the Southern neutrino sky; correlations with flaring sources, like micro-quasars and blazars; coincidences with GRBs; diffuse cosmic fluxes; correlation with gravitational waves; dark matter from the Sun, setting constraints in frameworks with neutrino and Kaluza-Klein particles; nuclearites, and magnetic monopoles.

\section{Acknowledgments}

The author acknowledges the support of the Spanish MICINN Consolider-Ingenio 2010 Programme under grant MultiDark CSD2009-00064 and of the Prometeo Programme of the Generalitat Valenciana.

\section{References}

[1] S. Adrián-Martínez et al., ANTARES Collaboration, Ap. J. 743, (2011) L14, [arXiv:1108.0292].

[2] S. Adrián-Martínez et al., ANTARES Collaboration, Accepted by Astropart. Phys., [arXiv:1111.3473].

[3] S. Adrián-Martínez et al., ANTARES Collaboration, Astropart. Phys. 35 (2012), 634-640, [arXiv:1110.2656].

[4] S. Adrián-Martínez et al., ANTARES Collaboration, JINST 7 (2012), T08002, [arXiv:astro-ph/1202.3894].

[5] S. Adrián-Martínez et al., ANTARES Collaboration, Phys. Lett. B714 (2012), 224-230, [arXiv: 1207.3105].

[6] S. Adrián-Martínez et al., ANTARES, VIRGO and LIGO Collaboration, [arXiv:1205.3018].

[7] S. Adrián-Martínez et al., ANTARES Collaboration, Submitted to Ap.J., [arXiv:1207.3105].

[8] J. A. Aguilar et al., ANTARES Collaboration, Astropart. Phys. 34 (2011) 539-549, [arXiv:1012.2204].

[9] J. A. Aguilar et al., ANTARES Collaboration, Phys. Lett. B696, (2011), 16-22, [arXiv:1011.3772].

[10] J. A. Aguilar et al., ANTARES Collaboration, Nuclear Instrum. Meth. in Physics Research, A 656 (2011), 11-38, [arXiv:1104.1607v1].

[11] V. Kulikovskiy, Neutrino 2012 Conference, Kyoto 2012.

[12] G. Pavalas, Proc. of the CSSP10 conference, Sinaia (2010), [arXiv:1010.2071v1].

[13] J.D. Zornoza, Dark matter searches with the ANTARES neutrino telescope: constraints to CMSSM and mUED models Proceedings of ICHEP2012, Melbourne, 2012. 